\title{
Sleep syncope: a new clinical entity or just a vasovagal syncope during wakefulness after sleep onset?
}

\author{
Philippe T. Gilchrist ${ }^{1} \cdot$ Federica Provini ${ }^{2,3}$ \\ Received: 28 December 2021 / Accepted: 28 December 2021 / Published online: 11 January 2022 \\ (c) The Author(s), under exclusive licence to Springer-Verlag GmbH Germany 2022
}

Keywords Nocturnal syncope $\cdot$ Neurally mediated syncope $\cdot$ Gastrointestinal $\cdot$ Loss of consciousness

Vasovagal syncope is conventionally defined as a neurally mediated reflex triggered by central hypovolemia, associated with vasodilation of arterial and venous beds and bradycardia. If the reticular activating system fails to receive a sufficient blood supply, loss of consciousness occurs [1]. Even mild reactions that do not reach the severity of loss of consciousness, including mild feelings that one is about to faint, can result in the avoidance of medical procedures and other wider public health implications. Namely, in addition to procedural complications in the medical setting and the risk of fall and injury caused by loss of consciousness, some patients with vasovagal syncope may also suffer from comorbidities, such as cardiac arrhythmias, structural cardiopulmonary disease, cerebrovascular disease, and neurological conditions, such as seizure, stroke, and migraine.

A detailed and structured medical history by an experienced clinician is the best approach to diagnosing vasovagal syncope, [2] even though some diagnostic testing is often required. Many factors can trigger vasovagal syncope, including orthostatic, blood loss, and situational factors such as emotional stress, dehydration, pain, and hyperventilation [3]. Moreover, there is a limited understanding of some of the physiological mechanisms and a lack of consensus regarding its origins and adaptive functions $[4,5]$. Even the term "vasovagal" also implies a mechanism that involves both vagal activity and vasodilation. This is a historically

Federica Provini

federica.provini@unibo.it

1 School of Psychological Sciences, Faculty of Medicine, Health, and Human Sciences, Macquarie University, North Ryde, NSW, Australia

2 Department of Biomedical and NeuroMotor Sciences (DiBiNeM), University of Bologna, Bologna, Italy

3 IRCCS, Istituto delle Scienze Neurologiche di Bologna, Bologna, Italy based definition and has been inconsistently applied. For example, the role of vagal activity has been challenged due to the ineffectiveness of atropine [6] to prevent the syncope, and because vasodilation and vasovagal symptoms may be preceded by withdrawal of sympathetic activity and sympathetically mediated active vasodilation [7, 8]. Given these complexities, developing clearer definitions is critical, including the possibility of establishing subtypes which may help improve the assessment and treatment of vasovagal syncope.

Sleep syncope is described as one possible subtype of vasovagal syncope interrupting sleep, causing abdominal symptoms, feeling faint, and loss of consciousness in bed or upon standing up [9-12]. While assuming the supine position is a common strategy recommended to some patients to prevent a vasovagal response, syncope is still possible in this position as observed during some invasive medical procedures, for example [9]. Similar to vasovagal syncope, sleep syncope requires a detailed description, corroborated by the spouse, and an exclusion of other possible causes [10]. A tilt-table test and follow-up assessments may also be recommended [13].

In this issue of Clinical Autonomic Research, Jardine and colleagues aimed to better understand the longer-term prognosis of sleep syncope. To do so, they conducted a prospective cohort study with a large sample of 1105 patients diagnosed with vasovagal syncope, including a subsample of 118 randomly selected patients without sleep syncope (classical vasovagal) and 69 with sleep syncope [10]. The study supported the hypothesis that the long-term prognosis of sleep syncope is good, despite the symptom severity. Further supporting the notion that sleep syncope is a possible form of vasovagal syncope, tilt-table results and mortality were also similar between both groups. Characteristic symptoms of sleep syncope included vagotonia and abdominal symptoms. Consistent with previous research, 
[11] blood-injection-injury phobia was more frequent in the sleep syncope patients when compared to classic vasovagal syncope. Overall, these findings offer a number of insights into this fascinating clinical entity, differentiating the phenomenon to some extent from vasovagal syncope, but it also raises some important questions for further research.

The symptoms of sleep syncope are not different from those of the conventional vasovagal syncope, other than the sleep interruption and the relative frequency of abdominal symptoms. Also, the term "sleep syncope" is somewhat of an oxymoron because consciousness cannot be lost during sleep. At the risk of being pedantic, it remains unclear whether sleep syncope is true syncope, or whether the experience is possibly better described as nocturnal syncope because the predominantly abdominal symptoms are upon wakening, and syncope can obviously only occur once conscious. Terminology aside, perhaps what makes the clinical entity of sleep/nocturnal syncope most fascinating is the currently largely unknown physiological mechanisms leading to waking, and the predominance of abdominal symptomatology. There is a case study of this phenomenon, including video recording, intermittent heart rate and blood pressure recordings, and one ECG recording [12]. Ideally, future research should include Holter monitoring and continuous blood pressure measurements during the sleep episodes, to begin to gain better insight into understanding the possible mechanisms and differentiating from other possible phenomena. However, these are difficult to obtain because of the rarity of sleep syncope. More comprehensive sleep studies would also be very important before establishing a new diagnostic category. Although firm inferences cannot be based upon the current findings, this appears to be a syndromic entity of high clinical interest.

The findings of Jardine and colleagues also raise important questions about the long-term prognosis of sleep syncope. The study initially ruled out many conditions and it will be very interesting for future studies to gather similar types of data at follow-up. It will be important to conduct a clinical workup to rule-out cardiac and other medical and psychiatric conditions, especially sleep disorders, mood disorders, and functional gastrointestinal disorders and other gastrointestinal conditions that might explain waking from sleep with abdominal symptoms [14]. Other conditions could account for a variety of sleep disturbances. Nonetheless, these possibilities do not make the current results any less interesting, and the possibility of a new clinical entity is likely.

A new diagnostic category of sleep or nocturnal syncope may help provide clarity to the future research and practice related to this interesting nocturnal phenomenon. Overall, while the differential diagnosis is complex in addition to multiple comorbidities, further evidence is needed to support this entity, including the clinical observation of this phenomenon, clarification of symptomatology (e.g., sleep vs. nocturnal), and an understanding of the mechanism behind the self-reported symptoms.

Funding None.

\section{Declarations}

Conflict of interest All the authors declare that they have no conflict of interest.

\section{References}

1. Folino AF (2007) Cerebral autoregulation and syncope. Prog Cardiovasc Dis 50:49-80

2. Wieling W, Kaufmann H (2021) What is the best method to diagnose a vasovagal syncope? Clin Auton Res 1:1-3

3. Moya A, Sutton R, Ammirati F, Blanc JJ, Brignole M, Dahm JB, et al (2009) Guidelines: guidelines for the diagnosis and management of syncope (version 2009): The Task Force for the Diagnosis and Management of Syncope of the European Society of Cardiology (ESC). Eur Heart J 30:2631

4. Alboni P, Alboni M (2017) Typical vasovagal syncope as a "defense mechanism" for the heart by contrasting sympathetic overactivity. Clin Auton Res 27:253-261

5. Diehl RR (2017) The target of vasovagal syncope is hemostasis and not heart protection. Clin Auton Res 27:215-217

6. Gerlach AL, Spellmeyer G, Vogele C, Huster R, Stevens S, Hetzel $\mathrm{G}$ et al (2006) Blood-injury phobia with and without a history of fainting: disgust sensitivity does not explain the fainting response. Psychosom Med 68:331-339

7. Gilchrist PT, Ditto B (2015) Sense of impending doom: Inhibitory activity in waiting blood donors who subsequently experience vasovagal symptoms. Biol Psychol 104:28-34

8. Halliwill JR, Dietz NM, Joyner MJ (1996) Active vasodilation during fainting: a hypothesis revisited. Clin Auton Res 6:233-236

9. Jardine DL, Krediet CP, Cortelli P, Wieling W (2006) Fainting in your sleep? Clin Auton Res 16:76-78

10. Jardine DL, Davis J, Frampton CM, Wieling W (in press) Sleep syncope: a prospective cohort study. Clinical Autonomic Research

11. Busweiler L, Jardine DL, Frampton CM, Wieling W (2010) Sleep syncope: important clinical associations with phobia and vagotonia. Sleep Med 11:929-933

12. Krediet CTP, Jardine D, Cortelli P, Visman AGR, Wieling W (2004) Vasovagal syncope interrupting sleep? Heart 90:e25-e25

13. de Jong JSY, Blok MRS, Thijs RD, Harms MPM, Hemels MEW, de Groot JR, van Dijk N, de Lange FJ (2020) Diagnostic yield and accuracy in a tertiary referral syncope unit validating the ESC guideline on syncope: a prospective cohort study. Europace 23:797-805

14. Cremonini F, Camilleri M, Zinsmeister AR, Herrick LM, Beebe T, Talley NJ (2009) Sleep disturbances are linked to both upper and lower gastrointestinal symptoms in the general population. Neurogastroenterol Motil 21:128-135 